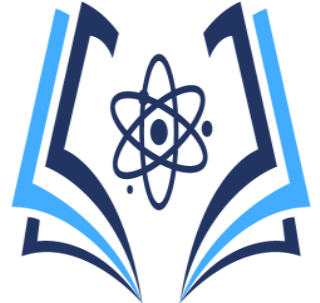

\title{
Los adolescentes y la educación en el uso de métodos anticonceptivos
}

\section{Adolescents and education in the use of contraceptive methods}

Uso de métodos anticonceptivos

\author{
Breeskin Natali Gutiérrez Izurieta ${ }^{(1)}$ \\ José Leonel Loor Bravo ${ }^{(2)}$ \\ Leyra Adriana Fonseca Liermo ${ }^{(3)}$ \\ Ana Maria Molina Santos, ${ }^{(4)}$ \\ ${ }^{1}$ Instituto Superior Universitario Portoviejo, Manabí, Ecuador. Email: natali151199@ @otmail.com \\ ${ }^{2}$ Instituto Superior Universitario Portoviejo, Manabí, Ecuador. Email: 1lloor2002@gmail.com \\ ${ }^{3}$ Facultad de Medicina General Calixto García. La Habana, Cuba, Email: leyadriana99@gmail.com \\ ${ }^{4}$ Facultad de Medicina General Calixto García. La Habana, Cuba, Email: molinasantosanamaria@gmail.com
}

Contacto: natali151199@ hotmail.com

\section{Resumen}

La investigación sobre embarazo precoz y educación en el uso de métodos anticonceptivos en adolescentes surge ante el carente o nulo conocimiento que poseen los adolescentes en salud sexual y reproductiva, cuyos aspectos son: enfermedades transmisión sexual, métodos anticonceptivos, planificación familiar, orientación sexual, entre otros aspectos, incluyendo los factores emocionales. Es por esta problemática y la necesidad de promover para la salud y bienestar de los adolescentes con intención de colaborar a que vivan una vida digna y estable hemos desarrollado este artículo, para acrecentar la importancia de enseñar en SSR, tomar medidas para prevenir las ETS, planificación familiar y métodos anticonceptivos con intención de que disminuya la cantidad de embarazos precoces y que la información que se les facilite sea de impacto en los adolescentes. El objetivo de este estudio fue: Educar a los adolescentes en torno a la prevención de embarazo precoz. Es un estudio descriptivo de corte transversal, realizado durante el semestre mayo-septiembre de 2020, con una muestra de 30 personas comprendidas entre 15 y 20 años de edad, todos dieron el consentimiento informado. Como resultado importante se logró identificar una minoría con escaso conocimiento en SSR. Se concluyó que es necesario que se implementen medidas para que los adolescentes tengan más facilidad al acceder a información sobre SSR y métodos de prevención para relaciones sexuales seguras. El hecho de que no lo consideren correcto a la edad de ellos, no significa que no exista curiosidad y lo puedan intentar sin prevenirse para las consecuencias.

Palabras clave: Embarazo precoz, métodos anticonceptivos, salud sexual y reproductiva.

\section{Abstract}

The research on early pregnancy and education in the use of contraceptive methods in adolescents arises from the lack or null knowledge that adolescents have 
in sexual and reproductive health, whose aspects are: sexually transmitted diseases, contraceptive methods, family planning, sexual orientation, among other aspects, including emotional factors. It is because of this problem and the need to promote the health and well-being of adolescents with the intention of helping them live a dignified and stable life, we have developed this article, to increase the importance of teaching SRH, take measures to prevent STDs, family planning and contraceptive methods with the intention of reducing the number of early pregnancies and that the information provided will have an impact on adolescents. The objective of this study was: To educate adolescents about the prevention of early pregnancy. It is a descriptive cross-sectional study, carried out during the MaySeptember 2020 semester, with a sample of 30 people between 15 and 20 years, all of whom gave informed consent. As an important result, it was possible to identify a minority with little knowledge in SRH. It was concluded that it is necessary to implement measures to make it easier for adolescents to access information on $\mathrm{SRH}$ and prevention methods for safer sex. The fact that adults do not considering it correct at their age does not mean that there is no curiosity and they can try without preventing themselves from the consequences.

Keywords: Early pregnancy, contraceptive methods, sexual and reproductive health.

\section{Introducción}

La educación sexual es un tema que es de gran relevancia abordar, conversarlo con los adolescentes, sin embargo, no se realiza una respectiva y correcta enseñanza, al contrario, es tratada como un tema que produce vergüenza o enojo según la persona que hable con otras, es decir, los padres se enojan porque en la actualidad se intenta expandir el alcance que tienen los educadores y que abarquen temas como este con sus estudiantes, así como otros sienten vergüenza para hablar del tema.
Publicado: Vol. 5, Nro. 2, 2021-12-31

"La adolescencia es la etapa comprendida entre los 10 y 19 años, es clasificada por la Organización Mundial de la Salud (OMS) en: temprana (10 a 13 años), media (14 a 16 años) y tardía (17 a 19 años). El embarazo en adolescentes es frecuente tanto en países desarrolla-dos como en vías de desarrollo y se considera un problema de salud pública por tener repercusiones adversas en la salud y a nivel social afectando a las madres, familiares y los niños" (Ñauta, García, Martínez, \& Ñauta, 2015)

Un embarazo precoz es el resultado del escaso o erróneo conocimiento que tienen los y las adolescentes respecto al tema de la educación sexual. Este problema se da a partir del momento en el cual los y las adolescentes atraviesan la etapa de la pubertad, en la cual empiezan a sentir los cambios de su cuerpo y sus hormonas empiezan a despertar nuevos intereses físicos en ellos, es así como éstos llamados por la curiosidad y el deseo de experimentación caen en sus ansias por hallar la respuesta física que sus cuerpos esperan y si no tienen conocimiento previo al respecto, el llevar a cabo prácticas sexuales de manera irresponsable podría acarrear problemas de salud y/o embarazos no deseados.

Actualmente es de gran asombro que todavía se den embarazos adolescentes, pues con la existencia de tantos métodos preventivos se creyera que no debería ser posible que sea tan recurrente el ver a adolescentes convirtiéndose en padres cuando aún no pueden cargar con la responsabilidad completa de sus propias vidas.

En países europeos es notoriamente sorprendente ver las bajas tasas de embarazos adolescentes, todo esto debido a la preparación y educación que dichos países otorgan a sus adolescentes, niñas y niños, puesto que la Educación Sexual es un conocimiento que se imparte como materia e incluso cuentan con centros de salud que les facilitan más conocimientos, métodos anticonceptivos, planificación familiar y 
hasta recursos como el aborto seguro sin prejuicios o maltratos por parte del personal médico.

Con el conocimiento obtenido a partir de este artículo se pretende informar a los jóvenes sobre educación sexual, concientizar que el tener hijos no es lo apropiado en la adolescencia, a tomar las medidas de anticoncepción y a quitar el prejuicio y temor que los padres y personas mayores han impuesto durante años, ya que en Latinoamérica la costumbre de pasar aquel erróneo y escaso conocimiento es el que ha llevado a los jóvenes a desconfiar de los métodos, prescindir de ellos y con ello obtienen enfermedades de transmisión sexual $\mathrm{u}$ obtienen un embarazo precoz, que claramente ninguno de los dos casos es deseado.

La OMS recomienda crear un centro de estas características por cada 100.000 jóvenes de entre 1425 años. La falta de continuidad y los errores en el uso de un método anticonceptivo parecen ser las principales causas de un embarazo inesperado en la adolescencia. Por ello debemos considerar la utilización de métodos de larga duración como uno de los más indicados a estas edades.

Es importante también combatir los falsos mitos que se han creado sobre los anticonceptivos hormonales (engordan, crean esterilidad), muy difundidos, que suponen un obstáculo en la utilización de un método altamente eficaz (Perpiñán, 2016).

Incluso el objetivo no solo sería llegar a los jóvenes que aún no son padres, sino a los que ya están a punto de tener un hijo y también llegar a los jóvenes que ya son padres, para que estos se tomen muy en serio las medidas de anticoncepción, que concienticen que la sobrepoblación es un problema real, que debemos detener.

El tener un hijo sin recursos suficientes para mantenerlos no es darles un buen vivir, como el Ecuador plantea en su Constitución. He ahí una de las importantes razones por las cuales debemos tener más control en la natalidad de niños y niñas, porque no es sólo el aspecto económico de los padres de los jóvenes que se ve afectado por un embarazo no deseado, también las vidas de dichos jóvenes cambian drásticamente y algunos, aunque ya sean padres, no maduran, por la misma razón de que son adolescentes, pues en sus jóvenes mentes ellos apenas están empezando a vivir, sin embargo ya se encuentran con el conflicto de ser padres consecuencia del escaso o nulo conocimiento de educación sexual que poseían.

Cabe mencionar que ser padre o madre sea malo, pero es causante de problemas para los jóvenes quienes deben enfocarse en realizar su carrera profesional, para desarrollarse como adulto funcional, para lo cual lo más ideal sería el no haber tenido hijos en la adolescencia o durante los primeros años de adultez. El objetivo de la investigación es: Educar a los adolescentes en torno a la prevención de embarazo precoz.

\section{Materiales y métodos}

La población fue de 30 personas divididas en 15 adolescentes masculinos y 15 adolescentes femeninas de la ciudad de Portoviejo, que fueron escogidos de manera intencional, el presente estudio es de corte transversal realizado durante el semestre Mayo-Agosto del 2020, las variables consideradas fueron: falta de conocimiento de educación sexual, métodos anticonceptivos, enfermedades de transmisión sexual, uso correcto de anticonceptivos, prevención de embarazo en jóvenes y la responsabilidad al tener relaciones sexuales.

Para lograr la realización del presente trabajo se han empleado materiales tecnológicos tanto como materiales tradicionales, a continuación, enlistamos los materiales tecnológicos de los cuales nos servimos para realizar nuestras encuestas como: laptop o computadora de escritorio, teléfonos celulares inteligentes, programa de Word, programa de Google Documentos para la realización de las encuestas, programa de Excel para la tabulación de 
resultados. Incluimos los materiales tradicionales de los cuales nos servimos para el proceso de recopilación de datos manuscritos como: cuadernos universitarios, esferos varios y notas adhesivas (PostIt).

\section{Resultados}

Los 30 encuestados (100\%) son adolescentes entre 15 y 20 años, ese factor coadyuvó a la calidad de la investigación facilitando el acceso a la información requerida.

Se ha logrado evidenciar que 23 de los encuestados (77\%) sí consideran que el embarazo en las adolescentes está causado principalmente por la falta de conocimiento sobre educación sexual, y el restante de 7 encuestados (23\%) consideran que las adolescentes quedan embarazadas por otros motivos o factores.

De acuerdo a la encuesta se logró analizar 30 adolescentes $(100 \%)$ de los cuales 26 adolescentes (87\%) si conocen sobre los métodos anticonceptivos para prevenir un embarazo y 4 adolescentes (13\%) no están conscientes sobre el uso de los métodos anticonceptivos para prevenir un embarazo.

De acuerdo a la investigación se estima que 21 adolescentes $(70 \%)$ están conscientes que el uso de métodos anticonceptivos nos protege de contraer enfermedades de trasmisión sexual, llevando a cabo que 9 adolescentes (30\%) afirman que están propensos al contagio de alguna enfermedad de trasmisión sexual aun usando los métodos anticonceptivos.

En cuanto a la educación sexual en el uso de los métodos anticonceptivos, los 30 adolescentes (100\%) afirmaron a ver recibida información requerida de cada uno de los métodos para prevenir un embarazo y para prevenir alguna enfermedad de trasmisión sexual.

En la investigación de los 30 adolescentes (100\%) 26 (87\%) de ellos tienen conocimiento sobre las consecuencias que trae las relaciones sexuales, como
Periodo. Julio - Diciembre 2021

Publicado: Vol. 5, Nro. 2, 2021-12-31

las enfermedades trasmisión sexual y las demás consecuencias, así como 4 adolescentes (13\%) no tienen idea alguna sobre las enfermedades de trasmisión sexual.

De acuerdo a la pregunta formulada y a los resultados, dan un porcentaje alto en la autoeducación en los adolescentes que han experimentado el acto sexual, 20 adolescentes (67\%) al tener su primera experiencia sexual, obtuvieron conocimientos positivos de las prevenciones que se deben tener, de los cuales 10 adolescentes (33\%) no adquirieron conocimientos y están propensos a tener un embarazo precoz o a alguna enfermedad de trasmisión sexual.

Teniendo en cuenta que hoy en día se habla mucho sobre la educación sexual, y dados los resultados de la investigación hay un cierto porcentaje en los adolescentes que no conocen sobre el uso correcto de algún método anticonceptivo, de los 30 adolescentes $21(70 \%)$ conocen sobre el uso correcto y 9 adolescentes (30\%) no tienen una de adecuada educación sobre el uso correcto de los métodos anticonceptivos.

Se evidenció según los resultados que 28 adolescentes (93\%) consideran que sí se deben impartir clases de educación sexual a la nueva generación de jóvenes, niñas y niños. Así como también $2(7 \%)$ de los adolescentes encuestados no lo consideran apropiado.

De acuerdo a los resultados que se obtuvieron, sabemos que 19 adolescentes (63\%) tienen conocimiento de algún otro método anticonceptivo, además del condón masculino, a la vez que 11 adolescentes (37\%) no saben de algún otro tipo de método anticonceptivo que no sea el condón masculino.

Finalmente, 7 adolescentes (23\%) afirman que la mujer, dentro de una relación es la única responsable del uso de métodos de prevención sexual. A diferencia de 23 adolescentes $(77 \%)$ que no 
consideran a la mujer como la única responsable del uso de preservativos dentro de una relación que involucre el acto sexual.

\section{Discusión}

El embarazo en adolescentes es un tema de muy relevante preocupación a nivel mundial, de manera especial en América Latina, cuyos países se sitúan con los números más altos en cuanto a embarazos adolescentes. En el actual artículo se presentan los resultados del análisis que se ha realizado respecto al embarazo en adolescentes y la educación en el uso de los métodos anticonceptivos en adolescentes.

"Unos 16 millones de muchachas de 15 a19 años y aproximadamente un millón de niñas menores de 15 años dan a luz cada año, la mayoría en países de ingresos bajos y medianos.

Las complicaciones durante el embarazo y el parto son la segunda causa de muerte entre las muchachas de 15 a19 años en todo el mundo

Los bebés de madres adolescentes se enfrentan a un riesgo considerablemente superior de morir que los nacidos de mujeres de 20 a 24 años"(Organización Mundial de la Salud, 2020)

Consideramos que una de las formas más efectivas de disminuir la cantidad de embarazos en adolescentes es por medio de la enseñanza del correcto uso de los métodos anticonceptivos, enseñar a las nuevas generaciones a no 'satanizar' el uso de los mismos en base a las doctrinas y costumbres de sus antecesores, quienes desde su época y más atrás vienen tratando el tema de la sexualidad como un tabú, es decir, como algo de lo que no se debe hablar y mucho menos enseñar, aunque sepan que sus hijos lo practican.

Algunas adolescentes planean y desean su embarazo, pero en muchos casos no es así. Los embarazos en la adolescencia son más probables en comunidades pobres, poco instruidas y rurales. En algunos países, los embarazos fuera del matrimonio no son raros. En cambio, algunas muchachas pueden recibir presión social para contraer matrimonio y, una vez casadas,
Periodo. Julio - Diciembre 2021

Publicado: Vol. 5, Nro. 2, 2021-12-31

para tener hijos. En países de ingresos medianos y bajos más del 30\% de las muchachas contraen matrimonio antes de los 18 años, y cerca del $14 \%$ antes de los 15 años.

Algunas muchachas no saben cómo evitar el embarazo, pues en muchos países no hay educación sexual. Es posible que se sientan demasiado cohibidas o avergonzadas para solicitar servicios de anticoncepción; puede que los anticonceptivos sean demasiado caros o que no sea fácil conseguirlos o incluso que sean ilegales. Aun cuando sea fácil conseguir anticonceptivos, las adolescentes activas sexualmente son menos propensas a usarlos que las mujeres adultas. Puede que las muchachas no puedan negarse a mantener relaciones sexuales no deseadas o a oponerse a las relaciones sexuales forzadas, que suelen ser sin protección (Organización Mundial de la Salud, 2020)

Durante el periodo de la elaboración de este artículo hemos hallado autores de trabajos similares con una perspectiva innovadora, relacionada a la que nos encontramos intencionados de compartir con la sociedad, en este caso (Gómez, Rodríguez, GómezSarduy, \& Torres, 2017), según los resultados de su investigación no hay intención hacia el uso de anticonceptivo en la práctica de relaciones sexuales entre los adolescentes. Esto habla a favor de que no visualizan el riesgo y de que son escasos deseos de usarlos en un futuro próximo. Resultados de otras investigaciones informan que no existe un método anticonceptivo ideal para uso en la adolescencia, como tampoco existen razones médicas para negar el uso de algunos basándose solo en razones de edad. El mejor método será aquel que la adolescente y, en el mejor de los casos, ambos miembros de la pareja, escojan, una vez que hubiesen recibido una completa y detallada información, y hayan sido interrogados exhaustivamente sobre cuestiones inherentes a su sexualidad y derecho reproductivo, lo que facilitará, 
finalmente, que se produzca el acuerdo entre el proveedor de salud y la pareja.

Deberá tomarse en consideración también a la hora de la selección del anticonceptivo, los hallazgos del examen físico general y de la esfera reproductiva, así como aspectos no médicos, pues estos no solo van a cumplir una función de prevención del embarazo, sino también la no menos importante de prevenir el contagio de una infección de transmisión sexual.

La actividad sexual en la adolescencia, específicamente las acciones educativas dirigidas a mejorar la percepción de riesgo del embarazo en adolescentes. Presenta relevancia para este artículo, el haber encontrado un nexo entre lo que hemos investigado y demostrado con resultados propios, que en la actualidad aún existe el pensamiento entre una cierta cantidad de adolescentes (en comparación con estudios anteriores de otros autores), ésta minoría no consideran importante el uso de los preservativos, haciendo denotar que este grupo de adolescentes no ha recibido o estado tomando enseñanza de acciones pertinentes respecto a lo que es salud sexual, con los resultados obtenidos de las encuestas, queda en evidencia que pese a que se ha tratado de crear consciencia respecto a las consecuencias de realizar actividad coital sin medidas de prevención, para evitar una enfermedad de transmisión sexual o embarazo no deseado, el número de adolescentes que mantenga aquel erróneo concepto respecto a la protección durante el acto sexual representa un grupo de personas al que debemos educar para la salud, corrigiendo de manera amable y pedagógica aquel problema. Los resultados no sólo arrojaron una minoría que necesita ser educada para su salud, pues satisfactoriamente se ha podido demostrar que en la actualidad son más los adolescentes que tienen conocimiento sobre preservativos, educación sexual, planificación familiar, responsabilidad compartida respecto a los métodos anticonceptivos, entre otros aspectos.
Es importante añadir que según (Mendoza, Claros, \& Peñaranda, 2016) los determinantes asociados al inicio temprano de la actividad sexual y embarazo entre adolescentes, entre ellos las inequidades, barreras de acceso a los servicios de salud sexual y reproductiva; y políticas en salud. Las consecuencias de estos dos fenómenos son graves para la salud pública, afectando las dimensiones familiares, social, de salud y económica. Si queremos impactar sobre la salud de los adolescentes, en especial sobre su salud sexual y reproductiva, se deben reducir estas inequidades, mejorar la accesibilidad de los adolescentes a los servicios de salud, estableciendo con ellos proyectos de vida a través del desarrollo de sus habilidades, brindar una adecuada educación sexual y mejorando el acceso a los métodos de prevención del embarazo y las enfermedades de transmisión sexual, esto viene a formar parte de lo que consideramos como los desaciertos que se han obtenido en la prevención de embarazos en adolescentes, pues (Castillo, 2016) ${ }^{[6]}$ afirma que las decisiones que toman los adolescentes acerca de su sexualidad se traducen en comportamientos con o sin riesgo para embarazo; dichas decisiones están mediadas por un proceso de razonamiento en el que confluyen las expectativas del adolescente, las expectativas de su pareja, la dinámica de su entorno social y su autoeficacia, entre otros factores psicosociales que influyen en la dimensión del ser y del saber hacer del adolescente; pero estos factores psicosociales no son considerados de manera relevante en las intervenciones para la prevención del embarazo en adolescente realizadas en Colombia.

Guiados por el estudio empírico y analítico hemos podido corroborar al igual que Castillo, que un tercio de las femeninas que se convierten en madres han deseado la maternidad en el momento en que se dio, lo que nos deja con un restante de una gran cantidad de mujeres que no se sentían preparadas para la maternidad y por aquel motivo, los resultados de las 
encuestas realizadas en Portoviejo nos dejan en claro que en su mayoría la actual generación de adolescentes, consideran pertinente que a la generación sucesora se le impartan clases de educación sexual, prevención y reproducción, para que aquellas historias no se repitan. Además en concordancia con (Tapia, Muñoz, \& Rosero, 2015) es realmente importante que los padres de los adolescentes sean su pilar de apoyo emocional y a la vez educacional, compartiendo conocimientos sobre reproducción sexual, y en caso de desconocerlos, lograr que sus hijos alcancen obtener dicho conocimiento por parte de personas académicamente preparadas para llevar a cabo la enseñanza, porque ni una sola madre adolescente se encuentra preparada para serlo, es un hecho que todas ellas reconocen, incluso muchas alegan no conocer sobre educación sexual o métodos anticonceptivos, así como otras madres adolescentes cuyo preservativo no funcionó, es importante que las personas que están en el entorno de éstas madres adolescentes las acompañen en el aspecto emocional.

De las consecuencias inmediatas que acarrea un embarazo en la adolescencia de acuerdo a (Loredo, Vargas, Casas, González, \& Gutiérrez, 2015), es indispensable considerar que las consecuencias del embarazo adolescente afectan a ambos miembros de la pareja, aunque probablemente es la mujer quien más las sufre. De ellas, destacan la fuerte carga emocional como consecuencia de su cambio físico y por la influencia, habitualmente negativa, del medio donde vive. En el cuadro II se describen las más frecuentes. Cuando ella informa a la familia de su embarazo, habitualmente es reprendida por sus padres $\mathrm{u}$ otros familiares, quienes la culpan de sentirse defraudados por su acción. De esta manera, es agredida emocional y, en ocasiones, físicamente. En algunos momentos también puede ser agredida por su pareja. Como consecuencia de su nueva condición, la adolescente puede presentar
Periodo. Julio - Diciembre 2021

Publicado: Vol. 5, Nro. 2, 2021-12-31 comportamientos poco habituales, destacando el descuido de su salud física y emocional, tales como infringirse daño físico e incluso provocarse el aborto. En concordancia con lo expuesto en el párrafo anterior, incluimos a (Torres, Zambrano, Jumbo, \& Brito, 2018) que con su estudio demostraron que los factores psicosociales representan uno de los principales causantes de embarazos en adolescente incrementando los costos de la nación debido a las tasas de mortalidad en abortos y entre otras complicaciones sociales y médicas que se presentan en aquellas mujeres embarazadas que no están edad apta para tener hijos. Lo que conlleva a la deserción escolar, ya que, los alumnos abandonan sus estudios a edades sumamente corta por tener que cumplir con las responsabilidades que acarrea la maternidad trayendo frustraciones a sus vidas e incrementando pérdidas en los ingresos de la nación que si se hubiese tomado o tratado de crear campañas de prevención de embarazos precoz. De éste dato podemos decir que nuestro conocimiento obtenido de manera empírica, confirma que ciertamente resulta más difícil para la adolescente femenina, que para el adolescente masculino atravesar las etapas de un embarazo precoz, lidiar con la maternidad como estilo de vida, adaptarse de manera financiera y el poder continuar con los estudios, así como también nuestro país gasta más en mantener una ciudadana adolescente y a su hijo, que realizando campañas de prevención de embarazos no deseados, incluso pueden mejorar la calidad de preservativos, en lugar de permitir que los adolescentes desconozcan sobre salud sexual y reproductiva.

Existe punto de coincidencia con varios autores respecto a los riesgos de salud que existen en los casos de embarazos en adolescentes El embarazo en este período tiene importantes consecuencias sobre la madre adolescente y su hijo. En la madre se destacan las hemorragias, el trabajo de parto prolongado, infección urinaria, bacteriuria asintomática, 
deserción escolar, afecciones placentarias, escasa ganancia de peso, con malnutrición materna asociada, parto prematuro, rotura prematura de las membranas ovulares, la incorporación temprana a la vida laboral, desajuste en la integración psicosocial, la escasa preparación para desarrollar una relación satisfactoria con los hijos, parto pretérmino, muerte materna, enfermedad hipertensiva, anemia, la incorporación temprana a la vida laboral, desajuste en la integración psicosocial, así como la escasa preparación para desarrollar una relación satisfactoria con los hijos, así como el aborto inducido. En el período del parto los problemas más frecuentes son las alteraciones en la presentación y en la posición del feto, que se relacionan con un desarrollo incompleto de la pelvis materna; lo que determina una incapacidad del canal del parto para permitir el paso del feto; estas distocias provocan aumento de los partos operatorios (fórceps y cesáreas). En los hijos se observan elevado riesgo de prematuridad, lesiones durante el parto, bajo peso al nacer, sepsis y muertes perinatales. Se ha confirmado que la mortalidad infantil en este grupo duplica o triplica la de los neonatos de madres mayores de 20 años. El embarazo en la adolescencia, además de constituir un riesgo médico elevado, lleva aparejado dificultades socioeconómicas y emocionales para la madre y su hijo(a), lo que se conoce como el "Síndrome del fracaso".

Los resultados en los que mayormente ha coincidido cada punto de discusión es en la ausencia o el escaso conocimiento sobre salud sexual y reproductiva en la que viven los adolescentes. En el caso del presente artículo, las encuestas nos dieron como resultado, que la población que fue escogida conocen de preservativos, la mayoría de ellos conocen uno distinto al condón masculino, mientras la minoría desconocen de algún otro método, acompañan a estos datos también el hecho de que una minoría no conocen el correcto uso de los profilácticos, así como
Publicado: Vol. 5, Nro. 2, 2021-12-31

tampoco consideran que el condón ayude a prevenir una ETS, esto es debido a que anteriormente no recibieron conocimientos sobre salud sexual y reproductiva, el desconocer ésta información puede llevar a embarazos no deseados en adolescentes, aunque sea una minoría, es importante para quienes nos dedicamos a educar para la salud y bienestar de las personas que adquieran conocimiento de este tipo. Pese a aquello, es alentador el saber que actualmente es mayor la cantidad de adolescentes que conocen de métodos anticonceptivos, de salud sexual, de reproducción humana consciente y que continuamente tratan de informarse por sus propios medios, debido a que el país aún no se preocupa lo suficiente como para colaborar en crear un área hospitalaria dedicada a la planificación familiar, educación sexual y reproductiva.

\section{Conclusiones}

El desconocer o saber poco sobre salud sexual y reproductiva afecta de manera considerable en la vida de los adolescentes, arriesgando su salud y su estabilidad. Los adolescentes que formaron parte de la investigación consideran de gran importancia que se enseñe a las futuras generaciones sobre salud sexual y reproductiva. También poner a consideración de darles una mejor accesibilidad a métodos anticonceptivos y de protección.

Se logró el objetivo propuesto satisfactoriamente, debido a que la mayoría de los encuestados tienen conocimiento sobre métodos anticonceptivos para prevenir un embarazo precoz, entre otros conocimientos sobre salud sexual y reproductiva.

Es la actual generación de adolescentes quienes consideran muy importante que el uso de métodos anticonceptivos o de protección no deba ser únicamente responsabilidad de la fémina, pues dentro de una relación de pareja, ambos acarrean la responsabilidad por partes iguales para prevenir enfermedades de transmisión sexual o un embarazo precoz. 


\section{Referencias Bibliográficas}

Ñauta, J., García, B., Martínez, B., \& Ñauta, J. (2015). Revista Médica HJCA. Obtenido de revistamedicahjca.iess.gob.ec:

http://revistamedicahjca.iess.gob.ec/ojs/index.php/HJCA/article/view/199/183

Perpiñán, C. M. (2016). Anticoncepción en adolescentes: Educación sexual y asistencia adecuada. Revista Española de Endocrinología Pediátrica.

Organización Mundial de la Salud. (31 de Enero de 2020). World Health Organization. Obtenido de www.who.int: https://www.who.int/es/news-room/fact-sheets/detail/adolescent-pregnancy

Gómez, R., Rodríguez, L.-M., Gómez-Sarduy, A., \& Torres, E. (Abril de 2017). Scielo. Obtenido de www.scielosp.org: https://www.scielosp.org/article/rcsp/2017.v43n2/180-190/es/\#

Mendoza, L., Claros, D., \& Peñaranda, C. (Junio de 2016). Scielo. Obtenido de scielo.conicyt.cl: https://scielo.conicyt.cl/scielo.php?pid=S0717-75262016000300012\&script=sci_arttext

Castillo, L. (08 de Octubre de 2016). Revista Científica Salud Uninorte. Obtenido de rcientificas.uninorte.edu.co: http://rcientificas.uninorte.edu.co/index.php/salud/article/view/8302/10422

Tapia, M., Muñoz, M., \& Rosero, J. (Mayo-Octubre de 2015). Repositerio UTN. Obtenido de repositorio.utn.edu.ec: http://repositorio.utn.edu.ec/bitstream/123456789/6172/2/06\%20ENF\%20714\%20ARTICULO.pdf

Loredo, A., Vargas, E., Casas, A., González, J., \& Gutiérrez, C. (20 de Noviembre de 2015). Redalyc. Obtenido de www.redalyc.org: https://www.redalyc.org/jatsRepo/4577/457750722016/457750722016.pdf

Torres, M., Zambrano, K., Jumbo, R., \& Brito, A. (22 de Mayo de 2018). Reciamuc. Obtenido de www.reciamuc.com: https://www.reciamuc.com/index.php/RECIAMUC/article/view/94/95

Favier, M.-A., Samón, M., Ruiz, Y., \& Franco, A. (Febrero de 2018). Revista de Información Científica. Obtenido de www.medigraphic.com: https://www.medigraphic.com/pdfs/revinfcie/ric-2018/ric181w.pdf

Rodríguez, N., Cala, Á., Nápoles, J., Milán, Y., \& Aguilar, M. (2017). Revista Información Científica. Obtenido de www.revinfcientifica.sld.cu: http://www.revinfcientifica.sld.cu/index.php/ric/article/view/27/53

Izaguirre-González, A., Aguilar-Reyes, V., Ramírez-Izcoa, A., Valladares-Rivera, G., Rivera-Mejía, W., Valladares-Rivera, E., . . . Fernández-Serrano, R. (2015). Dialnet UniRioja. Obtenido de dialnet.unirioja.es: https://dialnet.unirioja.es/servlet/articulo?codigo $=5797258$

Spain, P. (s.f.). Plan International ES. Obtenido de plan-international.es: https://plan-international.es/por-sernina/campana/embarazo-adolescente-0

Velasteguí, J., Hernández, M., Real, J., Roby, A., Alvarado, H., \& Haro, A. (Marzo de 2018). Scielo. Obtenido de scielo.sld.cu: http://scielo.sld.cu/scielo.php?script=sci_arttext\&pid=S0864-21252018000100005

Vera, M., \& Gómez, V. (08 de Noviembre de 2018). Revista Cognosis. Obtenido de https://186.46.160.238/index.php/Cognosis/index: https://doi.org/10.33936/cognosis.v3i5.1529 